\title{
Significato clinico della leucocitospermia
}

\author{
Rosita A. Condorelli ${ }^{1}$. Aldo E. Calogero ${ }^{1}$. Sandro La Vignera ${ }^{1}$
}

Accettato: 15 dicembre 2020 / Pubblicato online: 14 giugno 2021

(c) The Author(s) 2021

\section{Introduzione}

Il termine leucocitospermia identifica l'aumento della concentrazione seminale di leucociti nel liquido seminale oltre il valore soglia di $1 \mathrm{milione} / \mathrm{ml}$. Si tratta di un riscontro molto frequente nella pratica clinica, specialmente nell'infertilità maschile (fino al 30\%). L'atteggiamento del clinico risente della visione contrastante presente in letteratura riguardo l'importanza e le reali conseguenze che se ne attribuiscono [1]. La leucocitospermia rappresenta, da una parte, il parametro seminologico più rilevante per giustificare l'approfondimento microbiologico del campione seminale (anche se in molti casi non si accompagna a positività dell'esame colturale), dall'altra (specie nei casi di lieve rialzo delle concentrazioni seminali) un elemento funzionale alla migliore resa qualitativa della componente nemaspermica [2].

\section{Esame microscopico}

Nel seme vengono riscontrati in maniera predominante polimorfonucleati neutrofili che possono essere facilmente confusi con gli spermatidi multinucleati. A differenza degli altri tipi di leucociti (monociti, macrofagi, linfociti), i granulociti sono perossidasi-positivi [3]. Sebbene il cut-off sia stato posto a $1 \mathrm{mil} / \mathrm{ml}$, il danno che i leucociti sono in grado di apportare sugli spermatozoi è proporzionale all'aumento del rapporto leucociti/spermatozoi [3]. I leucociti, prevalentemente polimorfonucleati, vengono contati mediante

$\triangle$ R.A. Condorelli

rosita.condorelli@unict.it

1 Dipartimento di Medicina Clinica e Sperimentale, Università di Catania, Catania, Italia una procedura istochimica che identifica l'enzima perossidasi, caratteristico dei granulociti. Questa tecnica ha il vantaggio di essere di facile esecuzione, ma non identifica né $\mathrm{i}$ polimorfonucleati che hanno rilasciato i loro granuli, né altri tipi di leucociti come linfociti, macrofagi e monociti che non contengono perossidasi. Inoltre, possono essere differenziati dagli spermatidi e dagli spermatociti su uno striscio seminale colorato mediante tecnica di Papanicolau. La distinzione è basata sulle differenze nella colorazione, sulla grandezza e sulla forma del nucleo. I leucociti polimorfonucleati possono essere confusi con spermatidi multinucleati ma presentano una colorazione bluastra, in contrasto con la colorazione rosata degli spermatidi (Fig. 1).

Le linee guida in materia appaiono molto contrastanti; la Tabella 1 riassume le principali posizioni societarie in riferimento alla necessità di trattamento di tale condizione.

\section{Quali sono le domande che deve porsi il clinico dinnanzi a una condizione di leucocitospermia?}

\section{Si tratta di una condizione isolata o persistente?}

Non esiste accordo unanime sul concetto di persistenza; tuttavia, la conferma dell' aumento della concentrazione di leucociti ( $>1$ milione/ml) nel liquido seminale supporta almeno tre considerazioni di ordine pratico:

- esistenza di alterazioni morfostrutturali a carico della regione prostatica e/o prostato-vescicolare e/o epididimaria predisponenti (es. calcificazioni; aree di ectasia acinare) $[4,5]$;

- valutazione della presenza di anticorpi antispermatozoo come espressione della cronicizzazione di processo in- 
Fig. $1 P$, granulocita perossidasi positivo; $N$, cellula rotonda perossidasi negativa. Tratta da [3]

Tabella 1 Raccomandazioni diagnostiche delle principali società scientifiche internazionali. AUA, American Urologic Association; ASRM, American Society for Reproductive Medicine; CUA, Canadian Urological Association; EAU, European Association of Urology

\begin{tabular}{llll}
\hline & AUA e ASRM & CUA & EAU \\
\hline Raccomandazione diagnostica & $\begin{array}{l}\text { Si raccomanda la ricerca di } \\
\text { leucociti solamente in pazienti } \\
\text { con storia di infertilità inspiegata } \\
\text { o nel contesto di procedura di } \\
\text { fecondazione assistita }\end{array}$ & $\begin{array}{l}\text { In assenza di sintomi non si } \\
\text { raccomanda valutazione di possibile } \\
\text { infiammazione del tratto genitale }\end{array}$ & $\begin{array}{l}\text { La valutazione dei leucociti non } \\
\text { ènclusa nella valutazione } \\
\text { seminologica standard }\end{array}$ \\
\hline
\end{tabular}

fiammatorio locale in grado di innescare un'esaltata risposta immunitaria $[4,5]$;

- mancata eradicazione microbica $[4,5]$.

\section{Si associa a positivizzazione degli esami batterioscopici e colturali su liquido seminale?}

- Se sì, identificare possibili cause microbiche (batterica, protozoaria, parassitaria, micotica, virale) e rivalutare la concentrazione leucocitaria dopo eradicazione.

- Se no, considerare il possibile ruolo scavenger fornito dai leucociti nei riguardi degli elementi cellulari germinali immaturi (azione di immuno-sorveglianza).

- In presenza di apparente normalizzazione della concentrazione leucocitaria nel liquido seminale, ma in presenza di rialzo dei valori di $\mathrm{pH}$, iperviscosità e allungamento del tempo di liquefazione, considerare il possibile viraggio leuco-linfocitario meglio evidenziabile mediante tecniche di immunoistochimica e l'impiego di Ab monoclonali CD45 positivi panleucocitari.

\section{Si associa ad alterazione di parametri spermatici di secondo livello?}

L'associazione con aumento della percentuale di spermatozoi con DNA frammentato e/o l'aumento dei livelli seminali di marcatori di stress ossidativo identifica la presenza di una leucocitospermia non costituzionale.

\section{Si realizza nel contesto di un quadro di infertilità di coppia classificata come idiopatica?}

- Suggerire trattamento della leucocitospermia (FANS e/o corticosteroidi).

- Valutare associazione con parametri di secondo livello nel liquido seminale.

\section{Si associa ad altri criteri di Male Accessory Gland Infection/Inflammation (MAGI)}

- Anamnesi: storia di flogosi uro-genitali.

- Segni fisici: epididimo ispessito o dolente, anomalie all'esplorazione digito-anorettale.

- Caratteri del fluido prostatico: anomalie citologiche dopo massaggio prostatico.

- Caratteri eiaculatori: coltura con crescita significativa di batteri patogeni; viscosità e $\mathrm{pH}$ aumentati; anomalie biochimiche del plasma seminale.

\section{Conclusioni}

La leucocitospermia rappresenta una condizione di frequente riscontro nella pratica clinica. In molti casi non si accompagna a positività degli esami colturali, pur rappresentando spesso l'elemento di partenza nell'algoritmo diagnostico di eventuali indagini microbiologiche. Le linee guida in mate- 
ria appaiono discordanti riguardo le raccomandazioni diagnostiche. Le variabili da considerare nella pratica clinica sono differenti.

Funding Note Open access funding provided by Università degli Studi di Catania within the CRUI-CARE Agreement.

Conflitto di interesse Gli autori Rosita A. Condorelli, Aldo E. Calogero e Sandro La Vignera dichiarano di non avere conflitti di interesse.

Consenso informato Lo studio presentato in questo articolo non ha richiesto sperimentazione umana.

Studi sugli animali Gli autori non hanno eseguito studi sugli animali.

Nota della casa editrice Springer Nature rimane neutrale in riguardo alle rivendicazioni giurisdizionali nelle mappe pubblicate e nelle affiliazioni istituzionali.

Open Access This article is licensed under a Creative Commons Attribution 4.0 International License, which permits use, sharing, adaptation, distribution and reproduction in any medium or format, as long as you give appropriate credit to the original author(s) and the source, provide a link to the Creative Commons licence, and indicate if changes were made. The images or other third party material in this article are included in the article's Creative Commons licence, unless indicated otherwise in a credit line to the material. If material is not included in the article's Creative Commons licence and your intended use is not permitted by statutory regulation or exceeds the permitted use, you will need to obtain permission directly from the copyright holder. To view a copy of this licence, visit http://creativecommons.org/licenses/by/4.0/.

\section{Bibliografia}

1. Brunner RJ, Demeter JH, Sindhwani P (2019) Review of guidelines for the evaluation and treatment of leukocytospermia in male infertility. World J Mens Health 37:128-137

2. Ventimiglia E, Capogrosso P, Boeri L et al (2020) Leukocytospermia is not an informative predictor of positive semen culture in infertile men: results from a validation study of available guidelines. Hum Reprod Open 2020(3):hoaa039

3. World Health Organization (2010) Manual for the Examination and Processing of Human Semen, 5th edn. WHO Laboratory, Geneva

4. La Vignera S, Cannarella R, Aversa A et al (2020) Leukocytospermia in late adolescents: possible clinical interpretations. J Endocrinol Invest. https://doi.org/10.1007/s40618-020-01462-8

5. La Vignera S, Condorelli RA, Vicari E et al (2014) Microbiological investigation in male infertility: a practical overview. J Med Microbiol 63:1-14 